Acta Technologica Agriculturae 3

Nitra, Slovaca Universitas Agriculturae Nitriae, 2019, pp. 92-98

\title{
STOCHASTIC ANALYSIS OF MULTI-REACTION MODEL FOR NON-LINEAR THERMAL HISTORY
}

\author{
Alok DHAUNDIYAL ${ }^{1 *}$, Suraj BHAN SINGH ${ }^{2}$ \\ ${ }^{1}$ Szent István University, Doctoral School of Mechanical Engineering, Gödöllö, Hungary \\ ${ }^{2}$ Govind Ballabh Pant University of Agriculture and Technology, Pantnagar, India
}

\begin{abstract}
This paper investigates the effect of non-linear thermal profile on the numerical solution of the multi-reaction model. According to the practical perspective, the temperature distribution at a different section of pyrolysis reactor is not necessarily following the ideal thermal history; therefore, it is necessary to predict the behaviour of the system for the higher degree of freedom. TG thermogram is obtained by the thermal degradation of pine needles sample in the thermogravimetric analyser (TGA). The activation energy, frequency factor, reaction order and the scale, shape and location parameters of a stochastic function are estimated for the non-linear parabolic thermal profile. The conventional Laplace integral is used to approximate the multi-reaction model. Activation energy obtained for the non-thermal profile lies in the range of $57.5-60 \mathrm{~kJ} \cdot \mathrm{mol}^{-1}$, whereas the frequency factor varies from $10^{3}-10^{5} \mathrm{~min}^{-1}$. The obtained value of reaction order $(n)$ lies in the domain of $(0.9,1.6)$.
\end{abstract}

Keywords: biomass pyrolysis; kinetic parameters; the Laplace method; the multi-reaction model

Apart from the basic thermal applications of pyrolysis, certain other aspects of it must be addressed properly. For the same purpose, there is a need for a robust modelling scheme which can efficiently describe the pyrolysis phenomenon. At the elementary level, the thermal degradation of material (Li et al., 2009; Yan et al., 2009) (biodegradable or non-biodegradable) is performed by the thermogravimetry technique. This technique measures the extensive, as well as intensive properties of materials such as the mass, heat flux, and temperature variation of material with respect to the reference material. However, one of the most inherent parts of pyrolysis is the yield of secondary fuels, which can be further processed to get energetic biofuels. Therefore, the kinetic analysis of conversion process plays a major role in the determination of characteristics of the final products obtained from thermal degradation.

Method of assessing the kinetics of any process depends on the condensed phase reactions, as they guide the formulation of absolute rate theory without the involvement of momenta or ballistic motion. They signify position probability of particles (if the number of steps increases, probability follows the Gaussian distribution). Moreover, it also leads to an important approximation that activities of the reactants and products can be assumed to be one, or it would not be part of the equilibrium constant (Burnham, 2017). Mathematical schemes, iso-conversional and model-fitting methods, are used to simplify the kinetic mechanism of pyrolysis, but they have certain merit and demerit over each other. Inconsistent values of Arrhenius parameters and their hypothesis make them depart from the realistic overview of the problem. Chemical kinetics of material is determined by the reactivity of any given set of reactions. It becomes difficult to tackle a situation where one can compromise with the intrinsic behaviour of biomass pyrolysis. Therefore, there is a need to approximate the rate equation by using an asymptotic approach.

In this paper, the emphasis is laid on the effect of kinetic parameters on the approximated solution of the multireaction model. Several simplifications and approximations to the multi-reaction model are performed for an ideal thermal profile. Therefore, the heating rate is considered to vary with time. Furthermore, the effect of altering the sample temperature profile is also examined to determine the trend of the mass-loss curves. Thereafter, a comparative sketch of temperature non-linear variation is drawn against the results obtained from the isothermal and non-isothermal conditions.

\section{Material and methods}

\section{Mathematical modelling of biomass pyrolysis}

It has been assumed that the activation energies of concurrent reactions could be represented through the continuous normal distribution function (Anthony and Howard, 1976). However, the same model is tested for the different density functions, and it is reported that the modelling of biomass pyrolysis can be can be rather precisely described by the Rayleigh distribution than the Gaussian distribution (Dhaundiyal and Singh, 2018a).

Contact address: Alok Dhaundiyal, Szent István University, Doctoral School of Mechanical Engineering, Gödöllö, Hungary, e-mail: alok.dext@hotmail.com 
The Gamma and the Gaussian distributions, on the other hand, provide a good insight into the constant thermal history. A detailed study of assumption and restriction is reported in the literature (Dhaundiyal and Tewari, 2017). The material of interest is having a variety of reactivity; hence, it is reasonable to distinguish the set of independent parallel reactions by the differential equation for $i^{\text {th }}$ number of reactions. The solid-state reaction rate equation can be represented by Eq. 1:

$$
\frac{d\left(1-\alpha_{i}\right)}{d t}=-k_{i}(T) f\left(\alpha_{i}\right)
$$

where:

$k_{i}(T)$ - the rate constant, $\left(1-\alpha_{i}\right)$ denotes the remaining mass fraction

$t \quad-$ time

$f\left(\alpha_{i}\right)$ - the conversion function

The sum of the initial $\left(1-\alpha_{i}\right)$ should not be more than one. Parallel reaction model can be generalised from a set of discrete reactions to a continuous distribution. The final solution of differential equation (Eq. 1) is provided by Eq. 2. It is evident from Eq. 2 that the integral has no analytical solution; therefore, it requires some approximation schemes.

The mathematical expression of non-isothermal $n^{\text {th }}$ order multi-reaction model equation derived from the Eq. 1 is:

$$
(1-\alpha)=\left\{\begin{array}{c}
\int_{0}^{\infty} D(T) G(E) d E, \text { Firstorder }(n=1) \\
\int_{0}^{\infty}[1-(1-n) \ln (D(T))]^{\frac{1}{1-n}} G(E) d E, n^{\text {th }} \operatorname{order}(n \neq 1)
\end{array}\right.
$$

where:

$G(E)$ - the continuous distribution function

$n$ - represents the reaction order

$D(T)$ - the double exponential term

$T$ - temperature

$E\left(\mathrm{~kJ} \cdot \mathrm{mol}^{-1}\right)$ - denotes the activation energy.

To simplify the integral Eq. 2, the arbitrary unitless variable ' $z$ ' and the energy correction scale $y$ are assumed to:

$$
z_{0}=\frac{E}{R T_{0}} ;=\frac{E}{R\left(a l^{2}+T_{0}\right)} ; z_{m}=\frac{E}{R\left(a t^{2}+T_{0}\right)} \text { and } y=\frac{E}{\chi}
$$

where:

$$
\begin{array}{ll}
z_{0} \text { and } z_{m} & \begin{array}{l}
\text { - lower and upper limits of the double } \\
\text { exponential function }
\end{array} \\
R & - \text { a universal gas constant }
\end{array}
$$

$\chi\left(\mathrm{kJ} \cdot \mathrm{mol}^{-1}\right)$ - the threshold parameter

Eq. 2 denotes the distributed activation energy model equations for the first, as well as $n^{\text {th }}$ order reactions. It comprises two components. One of the components refers to the double exponential term $D(z)$, which is a function of non-linear thermal history encountered by the sample of biomass, whereas the other term denotes the continuous distribution function of varying activation energies of the overlapped concurrent reactions. To demonstrate the approach, the temperature depending term is simplified as Eq. 3:

$$
D(z)=\exp \left[\int_{z_{o}}^{z_{m}} \frac{A E}{\theta R} z^{-2} \exp (-z) d z\right]
$$

Deciding an appropriate density function should be accomplished with serious attention. In the stochastic modelling of biomass pyrolysis, density function represents the probabilistic behaviour of all those overlapped parallel reactions which differ in activation energies by a small margin. It is difficult to quote the superiority of one form over another, despite there being various other schemes proposed to describe the biomass with good accordance between experimental and predicted solution. The most common distribution function is Gaussian (Dhaundiyal and Tewari, 2017), but it is not necessary that thermo-analytical data follow the symmetrical pathway. There are also some other ways of analysing the problem of biomass pyrolysis (Dhaundiyal and Singh, 2017a). The function $G(E)$ is considered to follow three parametric forms (3-P) of Weibull distribution, where ' $\chi$ ' $\left(\mathrm{kJ} \cdot \mathrm{mol}^{-1}\right)$ represents the minimum barrier of the energy required to initiate the reactions. Here, $\mu$ and $\beta\left(\mathrm{kJ} \cdot \mathrm{mol}^{-1}\right)$ denote the shape and scale parameters of the Weibull distribution function respectively:

$$
G(y)=\frac{\mu}{\beta} \delta^{\mu-1}(y-1)^{\mu-1} e^{-\left((\delta(y-1))^{\mu}\right)}
$$

The practical value of dimension-less ratio $\delta=\frac{\chi}{\beta}$ is always $\delta>>1$.

The major drawback of the computational mechanism is to solve two distinct components simultaneously. Therefore, it requires a lot of resources to evaluate it for multiple times. In the previous approximation, Miura (1995) proposed the relationship for the effective disparity between activation energies of reactions by replacing the double exponential term with its approximations without paying heed to their influence on the value of the conversion $(\alpha)$ at the point of discord. His approach is latterly rebutted by Cai and Liu (2011). Assessment of Miura's approximation led to the miscellaneous value of frequency factor when the predicted solution is synchronised with the Gaussian distribution. Mathematically, with some assumption, the double exponential term can be approximated by the conventional Laplace scheme rather than the hit and trial methods. Hence, it is crucial to estimate the behaviour of the exponential term by demarcating the passive domain from the active ones.

To demonstrate the approach, ramping profile of temperature is assumed to follow the course of the parabolic temperature programme:

$$
T(K)=a l^{2}+T_{0}(K)
$$

where:

$I$ (min) - an instant of time

$a\left({ }^{\circ} \mathrm{C} \cdot \min ^{-2}\right)=\frac{1}{2} \frac{d \theta}{d t}-$ represents the rate of change of heating rate $(\theta)$. 
Boundary conditions for non-linear thermal history is given as:

$$
T(0)=T_{0} ; T(t)=T_{m}
$$

The expression for double exponential term is expressed as:

$$
D(z) \equiv \exp \left[\int_{z_{0}}^{z_{m}} \frac{A E e^{-z} d z}{2\left(z \sqrt{a z R\left(E-R z T_{0}\right)}\right)}\right]
$$

Applying the Laplace approximation to Eq. 6, we get:

$D(z) \equiv \exp \left(\frac{-A E}{2\left(z_{m} \sqrt{a z_{m} R\left(E-R z_{m} T_{0}\right.}\right)} e^{-z_{m}}\right)$ as $\frac{-E}{R\left(a t^{2}+T_{0}\right)} \rightarrow \infty$ (7)

It is obvious from Eq. 7 that the function $D(z)$ leaps from zero to one as $y$ increases by stepwise width of $y_{w}$ around the neighbourhood of the mean value of energy correction factor, $y_{s}$, thus it can be approximated through the Taylor series expansion about $y=y_{s}$ :

$$
J(y) \sim J\left(y_{s}\right)+\left(y-y_{s}\right) J^{\prime}\left(y_{s}\right)+\ldots
$$

where:

$$
(y)=\frac{y_{s}-y}{y_{w}}
$$

Using the following conditions, $J\left(y_{s}\right)=0$ and $J^{\prime}\left(y_{s}\right)=\frac{-1}{y_{w}}$ we get the values of:

$$
y_{s}=\frac{R T_{m}}{\chi} W(\tau) \text { and } y_{w}=\frac{R T_{m} y_{s}}{\left(\chi y_{s}+R T_{m}\right)} ; \tau=\frac{A}{\sqrt[2]{\frac{a\left(1-\frac{z_{m}}{z_{0}}\right)}{T_{m}}}}
$$

where:

$\tau \quad$ - a time scale factor

$W(\tau)$ - represents the Lambert $W$ function

Note: The ratio of $\frac{z_{m}}{z_{0}}$ lies in domain of $(0,1)$, therefore, $\tau$ will always be positive real number. Since $\frac{z_{m}}{z_{0}} \ll 1$, then $0<\left(1-\frac{z_{m}}{z_{0}}\right)$.

Eq. 2 demarcates two different set of reactions for the first and $n^{\text {th }}$ order reactions, hence they are solved and programmed separately. Thereafter, the mutually exclusive effect of them on the numerical solution is graphically demonstrated.

\section{Case 1: The first order reaction scheme}

All the parallel reactions that follow the first order reactions are represented by Eq. 2:

$$
1-\alpha=\int_{1}^{\infty} \mu \delta^{\mu}(y-1)^{\mu-1} D(z) e^{-\left\{(\delta(y-1))^{\mu}\right\}} d y
$$

Let $v=(y-1)$ be integral form transformed into:

$$
1-\alpha=\int_{1}^{\infty} \mu \delta^{\mu} v^{\mu-1} e^{-\left\{(\delta v)^{\mu}+D(z)\right\}} d v
$$

The term $S(v)=v^{\mu-1} e^{-\left\{(\delta v)^{\mu}\right\}}$ in Eq. 10 is assumed to be negligibly small everywhere except at the vicinity of $y_{s}$, therefore, it is to be evaluated by the Taylor expansion about $y=y_{s}$.

To demonstrate the approximation scheme of $n^{\text {th }}$ multi reaction model equations, application of Heaviside step function $H(x)$ is introduced to differentiate the distribution of activation energies for two different intervals of $x$.

After incorporating the step function $H(x)$, Eq. (10) can be represented in the form:

$$
\begin{gathered}
1-\alpha \sim \mu \delta^{\mu}\left[\left\{\left(v_{w}+1\right)\left(\int_{-\infty}^{\infty} e^{-\left\{e^{-x}-H(x)\right\}} S(v) d x\right)\right\}+\right. \\
\left.\left\{1-\left(\int_{0}^{v_{s}} G(v+1) d v\right)\right\}\right]
\end{gathered}
$$

Further simplification yields:

$$
\begin{aligned}
& 1-\alpha \sim \mu \delta^{\mu}\left[( v _ { s } ) ^ { ( \mu - 1 ) } \operatorname { e x p } ( - ( \delta v _ { s } ) ^ { \mu } ) ( v _ { w } + 1 ) \left\{I_{0}-\frac{\left(v_{w}+1\right)}{v_{s}}\right.\right. \\
& \left(\mu-\mu\left(\delta v_{s}\right)^{\mu}-1\right) I_{1}+\left(\frac{\left(v_{w}+1\right)}{v_{s}}\right)^{2} I_{2}\left(\mu^{2}\left(-3\left(\delta v_{s}\right)^{\mu}+\left(\delta v_{s}\right)^{2 \mu}+1\right)+\right. \\
& \left.3 \mu\left(\left(\delta v_{s}\right)^{\mu}-1\right)+2\right)-\left(\frac{\left(v_{w}+1\right)}{v_{s}}\right) I_{3}\left(\delta^{2} \mu^{4}\right)\left(-v_{s}^{2}\right)\left(\left(\delta v_{s}\right)^{\mu}-1\right)+ \\
& \mu^{3}\left(-\left(\delta v_{s}\right)^{2}-7\left(\delta v_{s}\right)^{\mu}+3\left(\delta v_{s}\right)^{2 \mu}+1\right)- \\
& \left.\left.\left.3 \mu^{2}\left(-6\left(\delta v_{s}\right)^{\mu}+\left(\delta v_{s}\right)^{2 \mu}+2\right)-11 \mu\left(\left(\delta v_{s}\right)^{\mu}-1\right)-6\right)\right\}+R\left(y_{s}\right)\right]
\end{aligned}
$$

Eq. 12 represents the required expression for the first order reactions.

The parameter $l_{i}$ is an unbiased parameter that needs to be computed once, so the first few computed values are:

$$
I_{0} \approx-0.5772, I_{1} \approx-0.9890, I_{2} \approx-1.8149, I_{3} \approx-5.8903
$$

To improve the accuracy of the given numerical solution, the other integral terms can be evaluated through integral equation:

$$
I_{j} \equiv \lim _{x \rightarrow \infty} \int_{-\infty}^{x} x^{j}\left(e^{-e^{-x}}-H(x)\right) d x j=0,1,2 \ldots
$$

\section{Case 2: The $n^{\text {th }}$ order reaction scheme}

Similarly, the numerical solution for $n^{\text {th }}$ order multi reaction model can be derived through the Binomial expansion of Eq. 13 as:

$$
(1-\alpha)_{n}=\int_{1}^{\infty}[\{1-(1-n) \ln (D(z))\}]^{\frac{1}{1-n}} G(y) d y
$$


Rearrangement and simplification of Eq. 13 yields the desirous expression for $n^{\text {th }}$ order reactions:

$$
\begin{aligned}
& (1-\alpha)_{n} \sim 1+\left[\left(\frac{n-5}{6}\right) R\left(y_{s}\right)-\left(v_{s}\right)^{(\mu-1)}\left(v_{w}+1\right) \exp \left(-\left(\delta v_{s}\right)^{\mu}\right.\right. \\
& \left\{\left(U_{0}-\frac{n}{2} v_{0}+\left(\frac{2 n-1}{6}\right) W_{0}\right)-\left(\frac{\left(v_{w}+1\right)}{W_{v_{s}}}\left(\mu-\mu\left(\delta v_{s}\right)^{\mu}-1\right)\right.\right. \\
& \left(U_{1}-\frac{n}{2} V_{1}+\frac{(2 n-1) W_{1}}{6}\right)+\left(( \frac { ( v _ { w } + 1 ) } { v _ { s } } ) ^ { 2 } \left(\mu^{2}\left(-3\left(\delta v_{s}\right)^{\mu}+\left(\delta v_{s}\right)^{2 \mu}+1\right)+\right.\right. \\
& \left.\left.3 \mu\left(\left(\delta v_{s}\right)^{\mu}-1\right)+2\right)\right)\left(U_{2}-\frac{n}{2} V_{2}+\frac{(2 n-1) W_{2}}{6}\right)-\left(\left(\frac{\left(v_{w}+1\right)}{v_{s}}\right)^{3}\right. \\
& \left(\delta^{2} \mu^{4}\left(-v_{s}^{2}\right)\left(\left(\delta v_{s}\right)^{\mu}-1\right)+\mu^{3}\left(-\left(\delta v_{s}\right)^{2}-7\left(\delta v_{s}\right)^{\mu}+3\left(\delta v_{s}\right)^{2 \mu}+1\right)-\right. \\
& \left.3\left(\delta v_{s}\right)^{2 \mu}+1\right)-3 \mu^{2}\left(-6\left(\delta v_{s}\right)^{\mu}+\left(\delta v_{s}\right)^{2 \mu}+2\right)- \\
& \left.\left.\left.11 \mu\left(\left(\delta v_{s}\right)^{\mu}-1\right)-6\right)\left(U_{3}-\frac{n}{2} V_{3}+\frac{(2 n-1) W_{3}}{6}\right)+\ldots\right\}\right]
\end{aligned}
$$

Eq. 14 represents the required expression for $n^{\text {th }}$ order reactions.

Here, the first few values of independent parameters $\left(U_{k^{\prime}}\right.$ $V_{k}, W_{k}$ ) are given below:

$$
\begin{gathered}
U_{0} \approx-0.3678, U_{1} \approx-0.2357, U_{2} \approx-0.1727, U_{3} \approx-0.1360, \\
V_{0} \approx-0.5676, V_{1} \approx-0.3515, V_{2} \approx-0.2525, V_{3} \approx-0.1964, \\
W_{0} \approx-0.6832, W_{1} \approx-0.4110, W_{2} \approx-0.2906, W_{3} \approx-0.2238
\end{gathered}
$$

\section{Modelling and simulation of prototype model}

MATLAB R2015a software is considered for the computation and simulation process. The non-linear thermal history experienced by the biomass sample is programmed for iterative loops. The objective functions of the computational problem are expressed by Eq. 12 and Eq. 14. Each allocated value goes through a common checkpoint for evaluating the acceptable range of permissible error. Until and unless the value qualifies itself as a suitable member of the permissible range, the iterative loop keeps looking for another assigned value to the input system. Eventually, thermo-chemical parameters are examined on the criterion of constraints imposed on the given pyrolysis problem. As the root mean square error and coefficient of regression is the foundation of grey box modelling, comparative tabulation of the nonlinear regime with linear ramping thermal history is given in Table 1.

\section{Application of pine waste}

Chemical analysis is performed through the CHNO-S analyser, whereas the higher heating value of pine waste is recorded with the help of a bomb calorimeter. Thermal evaluation of pine waste is based on thermogravimetricdifferential thermal analyser (Diamond TG/DTA, Perkin Elmer, and USA). The sample of $5.68 \mathrm{mg}$ is heated in a crucible pan of alumina from $303 \mathrm{~K}$ to $923 \mathrm{~K}$. To circumvent the obnoxious error of buoyant effect at the high temperature, horizontal differential type of prototype analyser is considered for experimentation. Thermocouple type ' $R$ ' is involved to measure the temperature inside the furnace. The volumetric flow rate of inert gas inside the chamber is fixed to $200 \mathrm{~mL} \cdot \mathrm{min}^{-1}$. Indium and tin are used as reference materials for the differential thermograms. Thermoanalytical data of pine needles sample is used for the qualitative assessment of multi-reaction model through a physically distinct regime of temperature. Elemental composition of pine needles is illustrated in Table 2.

Table 1 Comparative error of different non-isothermal profile

\begin{tabular}{|l||c|c|c|}
\hline Distribution type & Ramping profile (non-isothermal) & Root mean square error (RMSE) & $\boldsymbol{R}^{\mathbf{2}}$ (coefficient of regression) \\
\hline $\begin{array}{l}\text { Weibull } \\
\text { distribution }\end{array}$ & linear & 0.11 & 0.822 \\
\cline { 2 - 4 } & parabolic & 0.070 & 0.960 \\
\hline
\end{tabular}

Table 2 Elemental composition of pine needles

\begin{tabular}{|c|c|c|c|c|c|c|}
\hline $\mathbf{C} \%$ & $\mathbf{H} \%$ & $\mathbf{N} \%$ & $\mathbf{O} \%$ & $\mathbf{S} \%$ & Ash content $\mathbf{\%}$ ) & Higher heating value $\left(\mathbf{M J} \cdot \mathbf{k g}^{-\mathbf{1}} \mathbf{)}\right.$ \\
\hline \hline 53.7 & 5.21 & 0.61 & 32.13 & 0.22 & 4.72 & 19.5 \\
\hline
\end{tabular}

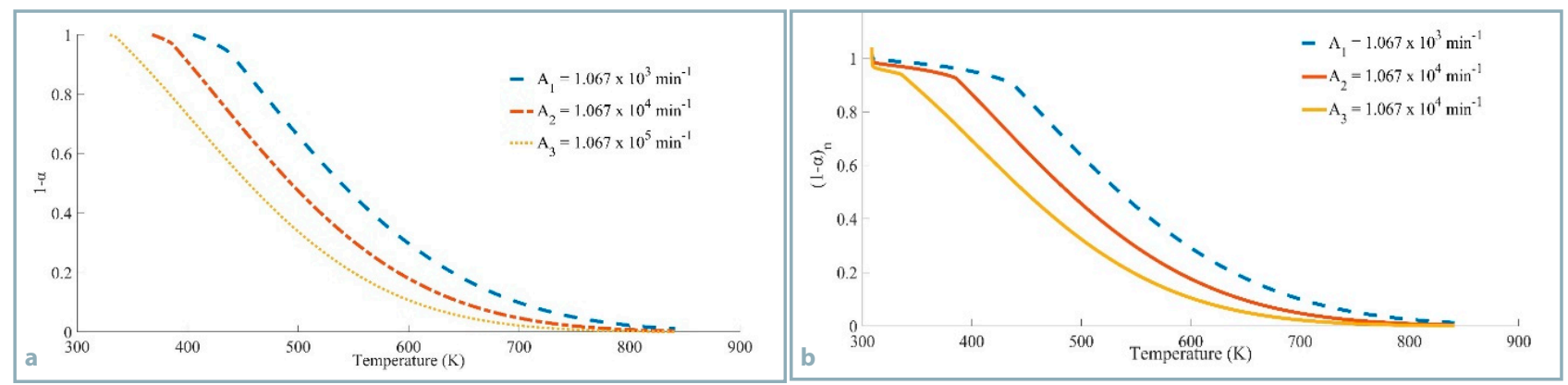

Fig. 1 The effect of frequency factor $(A)$ on the numerical solution

$\mathbf{a}-$ first order; $\mathbf{b}-n^{\text {th }} \operatorname{order}\left(\chi=1.10 \mathrm{~kJ} \cdot \mathrm{mol}^{-1} ; \mu=0.70 ; \beta=0.85 \mathrm{~kJ} \cdot \mathrm{mol}^{-1} ; T_{0}=290 \mathrm{~K} ; a=0.0135^{\circ} \mathrm{C} \cdot \mathrm{min}^{-2}\right)$ 


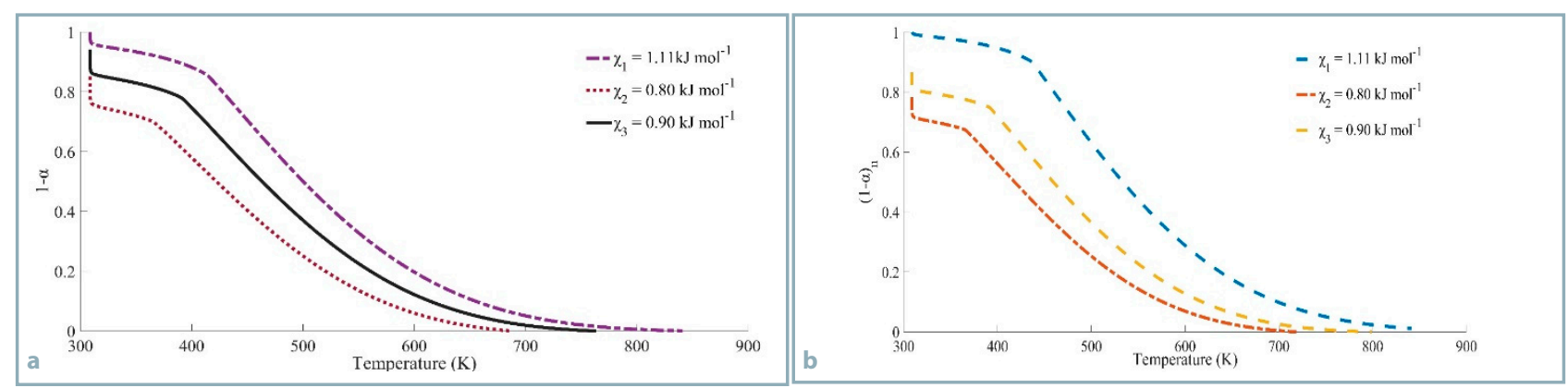

Fig. 2 The effect of location parameter of Weibull distribution on numerical solution $(\chi)$ $\mathbf{a}$ - first order; $\mathbf{b}-n^{\text {th }} \operatorname{order}\left(A=10^{3} \mathrm{~min}^{-1} ; \mu=0.70 ; \beta=0.85 \mathrm{~kJ} \cdot \mathrm{mol}^{-1} ; n=1.6 ; T_{0}=290 \mathrm{~K} ; a=0.0135^{\circ} \mathrm{C} \cdot \mathrm{min}^{-2}\right)$

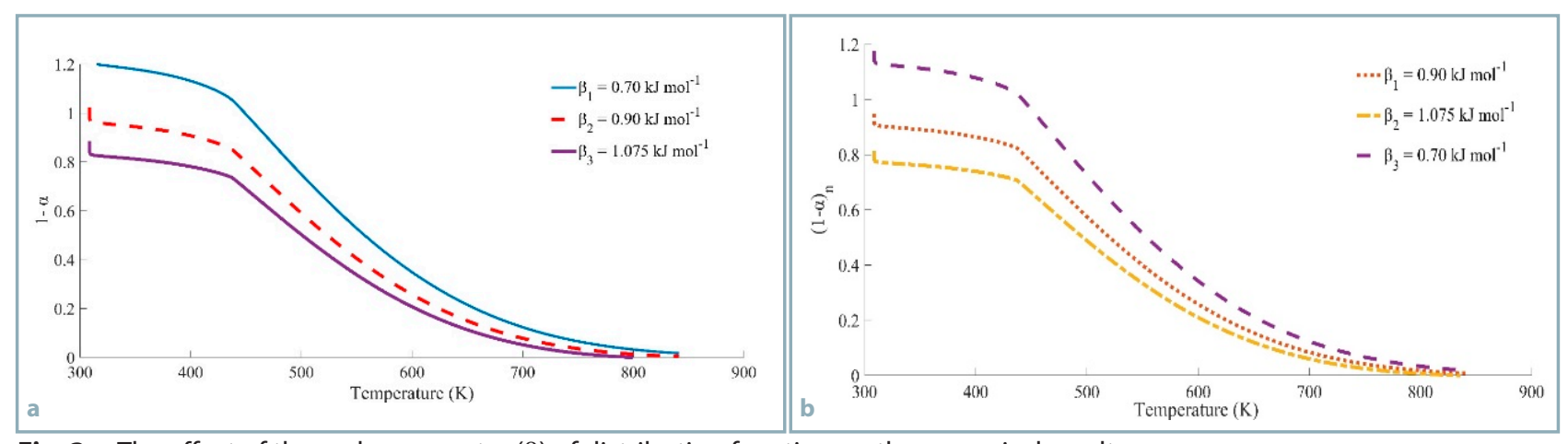

Fig. 3 The effect of the scale parameter $(\beta)$ of distribution function on the numerical result

$\mathbf{a}-$ first order; $\mathbf{b}-n^{\text {th }}$ order $\left(\chi=1.10 \mathrm{~kJ} \cdot \mathrm{mol}^{-1} ; \mu=0.70 ; A=10^{3} \mathrm{~min}^{-1} ; n=10 ; T_{0}=290 \mathrm{~K} ; a=0.0135^{\circ} \mathrm{C} \cdot \mathrm{min}^{-2}\right)$

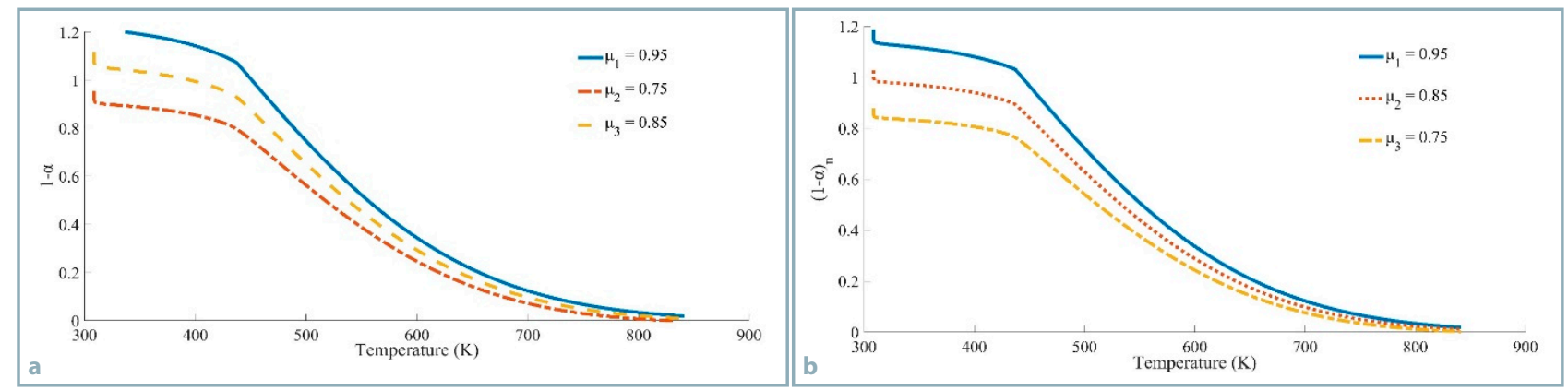

Fig. 4 The effect of the shape parameter $(\mu)$ of distribution function on the numerical result $\mathrm{a}$ - first order; $\mathrm{b}-n^{\text {th }}$ order $\left(\chi=1.10 \mathrm{~kJ} \cdot \mathrm{mol}^{-1} ; \beta=0.85 \mathrm{~kJ} \cdot \mathrm{mol}^{-1} ; A=10^{3} \mathrm{~min}^{-1} ; n=1.6 ; T_{0}=290 \mathrm{~K} ; a=0.0135^{\circ} \mathrm{C} \cdot \mathrm{min}^{-2}\right)$

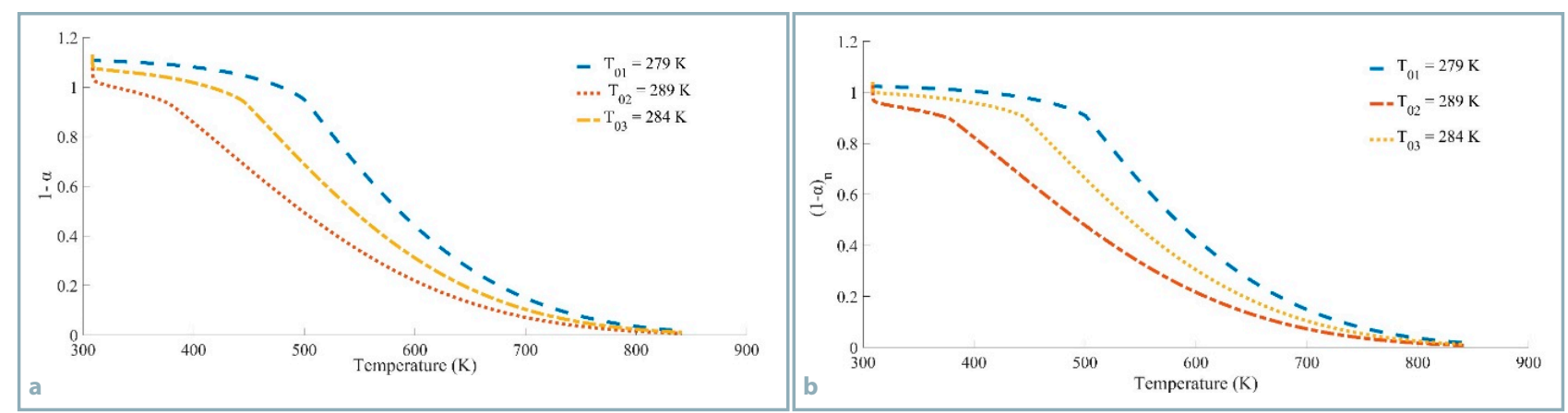

Fig. 5 The effect of initial temperature $\left(T_{0}\right)$ of furnace on the numerical result $\mathrm{a}-$ first order; $\mathrm{b}-n^{\text {th }} \operatorname{order}\left(\chi=1.10 \mathrm{~kJ} \cdot \mathrm{mol}^{-1} ; \beta=0.85 \mathrm{~kJ} \cdot \mathrm{mol}^{-1} ; A=10^{3} \mathrm{~min}^{-1} ; n=1.6 ; \mu=0.70 ; a=0.0135^{\circ} \mathrm{C} \cdot \mathrm{min}^{-2}\right)$ 


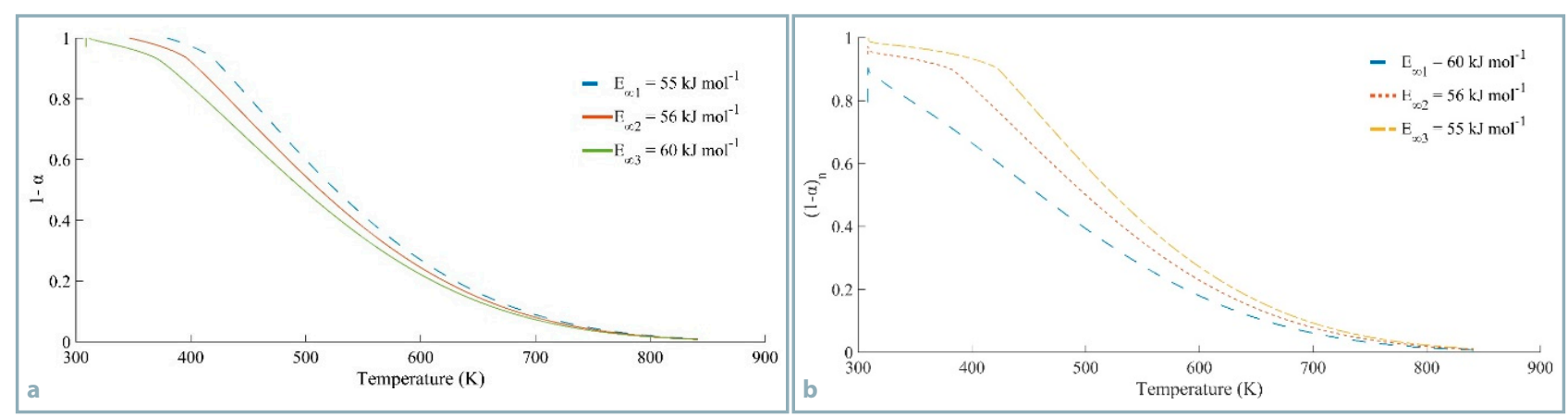

Fig. 6 The effect of upper limit of activation energy $\left(E_{\infty}\right)$ on the predicted result $\mathrm{a}$ - first order; $\mathrm{b}-n^{\text {th }} \operatorname{order}\left(\chi=1.10 \mathrm{~kJ} \cdot \mathrm{mol}^{-1} ; \mu=0.70 ; \beta=0.85 \mathrm{~kJ} \cdot \mathrm{mol}^{-1} ; T_{0}=290 \mathrm{~K} ; a=0.0135^{\circ} \mathrm{C} \cdot \mathrm{min}^{-2} ; A=10^{3} \mathrm{~min}^{-1}\right)$

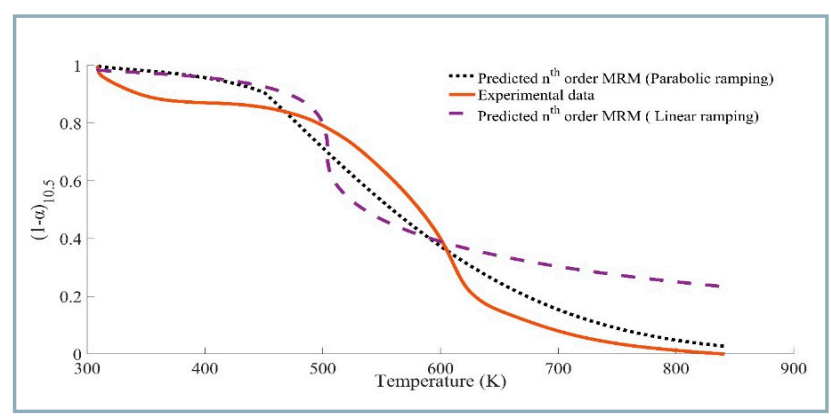

Fig. 7 Comparative sketch of experimental and predicted $n^{\text {th }}$ order pattern for non-linear thermal history

\section{Results and discussion}

The effects of various parameters on the pyrolysis problem are embedded together, and it is graphically illustrated. The effect of frequency factor $(A)$ for the non-linear regime is shown in Fig. 1a, b. At the onset of the pyrolysis process, the remaining mass fraction which is denoted by $(1-\alpha)$ is approximately equal to one and as temperature increases with time, it decreases unless it reaches its residual mass $\left(m_{r}\right)$. It seems that undulation in the remaining mass curves decreases as the decreasing trend in the frequency factor value has been encountered. Consequently, the curves get shifted in the right direction. Although the stochastic modelling through non-linear approach is perfectly depicting the initiating point of evaporation (the sunken segment of the curves), the rate of change of $(1-\alpha)$ with time is very subtle with the molecular collision of reactants. Therefore, the validation of the predicted model is suitable within the given range of $10^{3}<A\left(\mathrm{~min}^{-1}\right)<10^{5}$. If the value exceeds $10^{5}$, the condition of 'no-solution' arises as it is impossible for $(1-\alpha)$ to be negative or more than one unless the surface oxidation affects the chemical affinity of the pyrolytic reactions. On the other hand, the domain of frequency factor less than $10^{5}$ makes pyrolysis process slow, and the higher level of residual weight is obtained through the stochastic model, which overrides the possibilities of occurrence of proper devolatilization process. Observance for both cases (first-order, as well as $n^{\text {th }}$ order reactions) states the rate of conversion $(\alpha)$ decreases at a low value of $A$. The influence of variation of activation energy $\left(E_{\infty}\right)$ on the numerical solution is illustrated in Fig. $2 a$, b. The increase in the upper limit of activation energy causes shift of $(1-\alpha)$ curves to the left direction. The domain of activation energies for higher and the first order reactions has a variant effect on the shape, as well as thermochemical attributes for the non-linear regime of thermal decomposition. As the difference between activation energies of reactants gets wide up, the rate of conversion changes with time. For the activation energies of the first-order reactions $E_{\infty}>60 \mathrm{~kJ} \cdot \mathrm{mol}^{-1}$, the conversion of biomass is relatively faster than all those reactions that take place at $E_{\infty}>60 \mathrm{~kJ} \cdot \mathrm{mol}^{-1}$. Unlike the case of the first order, $n^{\text {th }}$ order reactions add up the concrete values to the outcome of the stochastic modelling. The interval of $55 \mathrm{~kJ} \cdot \mathrm{mol}^{-1}$ $\leq E_{\infty}<60 \mathrm{~kJ} \cdot \mathrm{mol}^{-1}$ gives a promising result to the numerical solution. Inequality holds good for the given experimental conditions, else the solution converges to the isothermal conditions of pyrolysis (Dhaundiyal and Singh, 2017b). The values obtained through stochastic modelling exhibit the compensatory effect amongst Arrhenius parameters, especially for activation energies (Burnham, 2014).

The effect of scale parameter $\beta\left(\mathrm{kJ} \cdot \mathrm{mol}^{-1}\right)$ of distribution function on the numerical solution is depicted in Fig. $3 a, b$. The domain of $\beta$ unanimously agreed for the higher and first-order reactions. The condition of no solution is imposed for all the values of $\beta \leq 0.70$, therefore, the defined domain of the scale parameter to get the desirous result is $0.90 \leq$ $\beta<1.075$. Unlike the Arrhenius parameters, the slope of conversion remains the same, but the temperature scale shifted up for the given domain of $\beta$.

The influence of the shape $\mu$ and the location parameters $\chi\left(\mathrm{kJ} \cdot \mathrm{mol}^{-1}\right)$ of distribution function on the numerical solution is depicted in Fig. 4a, b and Fig. 5a, b, respectively. Likewise, to the activation energies variation, the first and $n^{\text {th }}$ order reactions are inconsistent to each other. The magnitude of the shape parameters $\mu$ for $\mu \leq 0.85$ is asymptotically stable and provides solution to the experimental solution except for some infringement at the outbound of $(1-\alpha)$, however, the first-order reaction prediction defied the solution outright at $\mu \leq 0.85$. Therefore, the common solution for both reaction regimes lies in the domain of $0.75<\mu<0.85$. The value of the shape parameter causes the conversion $\alpha$ to increase drastically and shift of the $(1-\alpha)$ curves downward. On the other hand, the decrease in the location or threshold parameter initiates the pyrolysis process spontaneously, and the conversion $\alpha$ becomes independent of the temperature scale for a certain time. The domain of threshold parameter 
for the precise solution of the predicted solution varies from $0.90<\chi \leq 1.11$.

The relevance of physically different regime of thermal history and its effect on the predicted remaining mass proposition is depicted in Fig. 6a, b. With the increasing initial temperature $\left(T_{0}\right)$, the remaining mass fraction curves shifted towards the left, therefore, the temperature scale is reduced for the same conversion value. The behaviour of the curve changes rapidly to exponential decaying function as temperature elevates for the same initial time. The predicted solution converges swiftly at the vicinity of $284 \mathrm{~K}$, therefore, the initial experimental parameter $\left(T_{0}\right)$ must exist in an open interval of $284<T<289$.

On the other hand, the precise solution for the first order reactions is obtained for all the values of $T_{0}(K) \geq 289$. This implies that the first order reaction requires a hightemperature range to maintain the same conversion than that of the $n^{\text {th }}$ order reactions at the same instance of time I (min). At the onset of pyrolysis process, the inflexion points are visible, which demarcates evaporation of water content in the biomass, but as the temperature decreases, the inflexion point changes at the same time for different initial temperature $\left(T_{0}\right)$. The domain of ' $a$ ' for the first- order reaction suggests the numerical solution $\forall a<0.014^{\circ} \mathrm{C} \cdot \mathrm{min}^{-2}$.

It is worth mentioning that quantitative analysis of linear ramping problem (Dhaundiyal et al., 2019; Dhaundiyal et al., 2018b) of non-linear thermal history provided the qualitative insight of the same problem through better fitting of predicted model to the given thermo-analytical data (Table 1). It is observed through linear ramping that the activation energy is increased for the same experimentation when the linear thermal history is executed, therefore it is sure that frequency factor and activation energy vary simultaneously with thermal history. Sensitivity and attributes of curves largely rely upon scale, threshold and shape parameters.

\section{Conclusion}

Through predicted data of non-linear thermal history, $57.5 \mathrm{~kJ} \cdot \mathrm{mol}^{-1}$ can be incorporated as the upper limit of activation energies, which is a bit lower than the expected ones (Dhaundiyal and Singh, 2018a) due to the mutually compensatory effect. On the other hand, the frequency factor lies in the range of $10^{3}$ to $10^{5} \mathrm{~min}^{-1}$. The initial temperature should be more than $289 \mathrm{~K}$. The value of ' $a$ ' lower than $0.014^{\circ} \mathrm{C} \cdot \mathrm{min}^{-2}$ violates the boundary condition, therefore the value of $0.014{ }^{\circ} \mathrm{C} \cdot \mathrm{min}^{-2}$ is considered as the minimum permissible value of the parameter ' $a$ '. The value lower than $11 \mathrm{~kJ} \cdot \mathrm{mol}^{-1}$ exhibit $(1-\alpha)$ curve becomes asymptotic to the isothermal lines. The reaction order should be within the domain of $0.9<n<1.6$ for numerical prediction of the non-linear ramping profile. The derived results provided good information about variation of the heating rate with time and the effect of initial temperature on the different reaction orders.

\section{References}

ANTHONY, D. B. - HOWARD, J. B. 1976. Coal devolatilization and hydrogastification. In Journal of American Chemical Society, vol. 22, no. 4, pp. 625-656.

BURNHAM, A. K. 2017. Introduction to chemical kinetics. In Global Chemical Kinetics of Fossil Fuels, pp. 25-74.

BURNHAM, A. K. 2014. Obtaining reliable phenomenological chemical kinetic models for real-world applications. In Thermochimica Acta, vol. 597, pp. 35-40.

CAl, J. M. - Li, T. - LIU, R. A. 2007. A critical study of the Miura-Maki integral method for the estimation of the kinetic parameters of the distributed activation energy model. In Journal of Bioresource Technology, vol. 102, pp. 3894-3899.

DHAUNDIYAL, A - SINGH, S. B. - ATSU, D. - DHAUNDIYAL, R. 2019. Application of Monte Carlo simulation for energy modelling. In Journal of American Chemical Society (Omega), vol. 4, no. 3, pp. 4984-4990.

DHAUNDIYAL, A. - B. SINGH, S. 2018a. Mathematical insight to non-isothermal pyrolysis of pine needles for different probability distribution functions. In Biofuels, vol. 9, no. 5, pp. 647-658.

DHAUNDIYAL, A. - SINGH, S. B - HAMON, M. 2018b. Study of distributed activation energy model using bivariate distribution function, $f\left(E_{1}, E_{2}\right)$. In Thermal Science and Engineering Progress, vol. 5, pp. 388-404.

DHAUNDIYAL, A. - SINGH, S. B. 2017a. Asymptotic approximations to the isothermal pyrolysis of deodara leaves using gamma distribution. In Journal of Universitas Scientiarum, vol. 22, no. 3, pp. 263-284.

DHAUNDIYAL, A. - SINGH, S. B. 2017b. Approximations to the non-isothermal distributed activation energy model for biomass pyrolysis using the Rayleigh distribution. In Acta Technologica Agriculturae, vol. 20, no. 3, pp.78-84.

DHAUNDIYAL, A. - TEWARI, P. 2017. Kinetic parameters for the thermal decomposition of forest waste using distributed activation energy model (DAEM). In Environmental and Climate Technologies, vol. 19, no. 1, pp. 15-32.

LI, Z. - LIU, C. - CHEN, Z. - QUAIN, J. - ZHAO, W. - ZHU, Q. 2009. Analysis of coals and biomass pyrolysis using the distributed activation energy model. In Bioresource Technology, vol. 100, no. 2, pp. 948-952.

MIURA, K. 1995. A new and simple method to estimate $f(E)$ and $k o(E)$ in the distributed activation energy model from three sets of experimental data. In Journal of Energy and Fuels, vol. 9, no. 2, pp. 302-307.

YAN, J. H. - ZHU, H. M. - JIANG, X. G. - CHI, Y. - CEN, K. F. 2009. Analysis of volatile species kinetics during typical medical waste materials pyrolysis using a distributed activation energy model. In Journal of Hazardous Materials, vol. 162, no. 2-3, pp. 646-651. 